\title{
Antihypertensive Medications and Change in Stages of Chronic Kidney Disease
}

\author{
Marina Komaroff ${ }^{D},{ }^{1}$ Fasika Tedla, ${ }^{2}$ Elizabeth Helzner, ${ }^{1}$ and Michael A. Joseph ${ }^{1}$ \\ ${ }^{1}$ School of Public Health, Department of Epidemiology and Biostatistics, State University of New York Downstate Medical Center, \\ Brooklyn, NY, USA \\ ${ }^{2}$ College of Medicine, Department of Medicine, State University of New York Downstate Medical Center, Brooklyn, NY, USA
}

Correspondence should be addressed to Marina Komaroff; genmar2k@gmail.com

Received 3 August 2017; Revised 19 January 2018; Accepted 28 January 2018; Published 25 February 2018

Academic Editor: Katarzyna Zorena

\begin{abstract}
Copyright (C) 2018 Marina Komaroff et al. This is an open access article distributed under the Creative Commons Attribution License, which permits unrestricted use, distribution, and reproduction in any medium, provided the original work is properly cited.

Objectives. The goal of this study is to estimate the change in the relationships between use of five classes of antihypertensive medications and stages of Chronic Kidney Disease (CKD) in American adults treated for hypertension. Methods. The US National Health and Nutrition Examination Survey (NHANES) data sets 1999-2012 were used with the final analytical sample of 3,045 participants. Population prevalence estimates were calculated using the NHANES survey design weights. Inferential analyses were done with binomial logistic regression models. Results. The odds of advanced (3, 4, and 5 combined) versus early CKD stages ( 1 and 2 combined) were significantly higher among patients treated with Angiotensin Receptor Blockers (ARB) versus those not treated with ARB in 2009-2012 (adjusted odds ratio (95\% confidence interval) $=2.52(1.32-4.80)$ ). From 1999 to 2012 , the increase in this relationship was significant $(p=0.0023)$ for users of ARB polytherapy and in users of ARB in patients with albuminuria $(p=0.0031)$. Conclusion. Aggressive pharmacological management of hypertension with ARB as add-on therapy may have accelerated kidney damage in American adults. However, prospective longitudinal studies are needed to establish proper temporal sequence in this relationship.
\end{abstract}

\section{Introduction}

Hypertension (HTN) is the second leading cause of kidney failure [1-7]. Thus, effective treatment of hypertension for American adults with CKD to slow the progression of the disease is important. In the Seventh Report of the Joint National Committee on Prevention, Detection, Evaluation, and Treatment of High Blood Pressure (JNC 7, 2003), Angiotensin Converting Enzyme (ACE) Inhibitors or Angiotensin II Receptor Blockers (ARBs) were recommended as initial or add-on pharmacological therapy particularly for patients with CKD $[5,7]$. There has been a considerable increase in treatment and control of hypertension with antihypertensive medications in patients with CKD since release of JNC 7, 2003 [8-14]. Recent studies suggest a potential relationship between use of antihypertensive medications and deterioration in kidney function $[15,16]$. The major point was that antihypertensive agents to control blood pressure may also impact kidney function independently of their effect on blood pressure [6].

This study examines the relationships between use of antihypertension medications and CKD stages among American adults (18 years or older) treated for hypertension and determines whether these relationships have changed from 1999 to 2012 (stacked cross-sectional design). There was public health campaign during this time period to aggressively treat and lower blood pressure particularly by use of ACE I and ARBs in patients with CKD. We hypothesized that if the recommended treatment of hypertension in patients with CKD worked as intended then the associations with stages of CKD should be moving towards a decrease in the prevalence of advanced stages or at least stay stable; otherwise, the recommended therapy might appear to be more harmful than beneficial and should perhaps be revised. 


\section{Material and Methods}

2.1. Database Overview. The US National Health and Nutrition Examination Survey (NHANES) is a representative sample of noninstitutionalized US civilian residents that are currently conducted every 2 years. NHANES data are obtained using a complex, multistage, cluster, and probability sampling design that incorporates differential probabilities of selecting participants. The data collection, reporting, and analyses followed consistent definitions that can be found on the websites [17].

2.2. Study Design. Three time points (NHANES cycles 1999-2004, 2005-2008, and 2009-2012) were utilized to determine if these relationships have changed over time (stacked cross-sectional design).

2.3. Study Population. Inclusion criteria were participants 18 years of age or older, who completed questionnaire and underwent a physical examination, reported demographic information (age, gender, and race), provided self-reported information on hypertension including treatment, had blood pressure measured, had estimated glomerular filtration rate (eGFR) and urine albumin/creatinine ratio (ACR), and were not pregnant if female. Population of interest was participants with hypertensive CKD, who satisfied inclusion criteria, and were treated for hypertension.

2.4. Definitions. CKD was defined by the level of glomerular filtration rate (GFR) and the urine albumin-to-creatinine ratio (ACR) $[6,18]$. The stages of $\mathrm{CKD}$ and albumin to creatinine ratio (ACR) were classified following the National Kidney Foundation (NKF) Kidney Disease Outcomes Quality Initiative (KDOQI) $[6,18]$.

Hypertension for participants with CKD was present if systolic blood pressure is greater than or equal to $130 \mathrm{~mm} \mathrm{Hg}$ or diastolic blood pressure greater than or equal to $80 \mathrm{~mm} \mathrm{Hg}$, or subject currently was taking medication to lower high blood pressure $[7,19]$.

Treatment of high blood pressure was defined by an affirmative response to the question "Have you ever been told by a doctor or health professional that you had hypertension, also called high blood pressure?" and to the following questions: "Because of your high blood pressure/hypertension, have you ever been told to take prescribed medicine?" and "Are you now following this advice to take prescribed medicine?" $[7,19]$.

Diabetes was present if glycohemoglobin (HbAlc) was equal to or greater than $6.5 \%$ [20].

The five antihypertensive classes of medications were (1) ACE I (Angiotensin Converting Enzyme Inhibitors); (2) ARB (Angiotensin Receptor Blockers); (3) BB ( $\beta$-blockers); (4) CCB (Calcium Channel Blockers); and (5) Diuretics [5, 7]. Monotherapy was defined when participates reported use of only one antihypertensive drug, and polytherapy which is the same as combination therapy was defined when participants reported use of more than one antihypertensive agent. When single pill of antihypertensive combination was reported, it was classified as polytherapy.
Data from seven NHANES surveys 1999-2012 included 71,916 records. The analytical sample of hypertensive participants with CKD who were treated for HTN and satisfied inclusion criteria was 3,045; see consort flow diagram, Figure 1.

2.5. Statistical Analyses. The prevalence of CKD by classes of antihypertensive medications was age-standardized to the 2000 US standard population using 4 age groups [19, 21]. Variance estimation was performed utilizing Taylor series method. Relative standard errors (RSE) were calculated, and those greater than $30 \%$ were considered potentially statistically unreliable per NHANES Analytic Guidance and marked with the asterisk $(*)$ in the tables [21]. Because of small sample size of severe advanced stages ( 4 or 5 ), the main analyses were focused on CKD early stages 1 and 2 combined and advanced stages 3,4 , and 5 combined.

2.5.1. Model Description. Binomial logistic regression models were used to test the hypotheses of the study. In the models for three time points (NHANES cycles 1999-2004, 2005-2008, and 2009-2012), the ordinal coded dichotomous variable for stages of CKD was the dependent variable (outcome), time was independent variable, and class of antihypertensive medication was the independent variable (effect modifier). Interaction terms (time $\times$ class of medication) or (time $\times$ mono- or polytherapy) were used to test the null hypothesis of no difference in change in the odds ratios for patients treated versus untreated with particular class of medication over 14-year time period. The models included the following covariates: age, gender, race/ethnicity, body mass index (BMI), smoking status, diabetes, and albuminuria to adjust for potential confounding. The models accommodated the complex multistage sample survey design following NHANES Analytic and Reporting guidance [21]. To preserve Type I error in multiple subgroup analyses, the Bonferroni correction $\alpha / k$ was used where $\mathrm{k}$ is the number of analyses.

2.5.2. Trend Analyses. Trend was determined with the statistically significant interaction between class of medication and time (ordinal variable) indicating that their relationships had changed over time. In the models, the outcome variable was modeled as Logits that contrasted advanced (3, 4, and 5 combined) with the earlier ( 1 and 2 combined) stages of CKD. The odds ratios (95\% Confidence Interval) were reported for each of five classes of medications: ACEI, ARB, BB, CCB, DIU, and poly- or monotherapy.

Exploratory Analyses. Subgroup analyses were performed with the same model and covariates for the following groups: diabetes (absent/present); albuminuria/ACR $>30 \mathrm{mg} / \mathrm{g}$ (absent/present); microalbuminuria/ACR 30-<300 mg/g (absent/present); macroalbuminuria/ACR $>300 \mathrm{mg} / \mathrm{g}$ (absent/ present); diabetes with albuminuria; diabetes with microalbuminuria; diabetes with macroalbuminuria; diabetes without albuminuria; diabetes without microalbuminuria; diabetes without macroalbuminuria; no diabetes and albuminuria; no diabetes and microalbumin-uria; no diabetes and macroalbuminuria. 


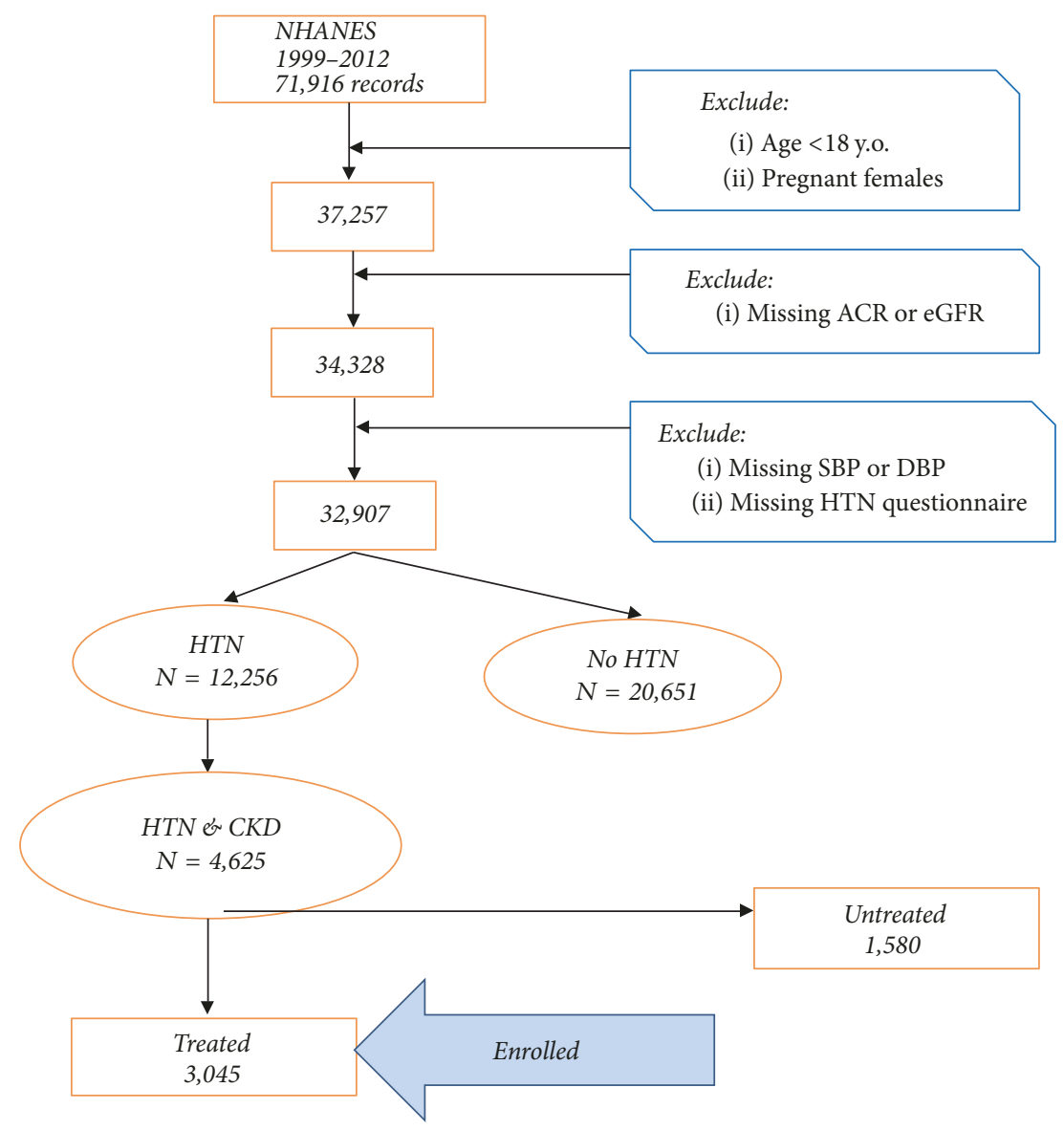

FIGURE 1: Consort flow diagram.

Statistical analyses were conducted using the SAS ${ }^{\circledR}$ System for Windows (release 9.3; SAS ${ }^{\circledR}$ Institute Inc., Cary, NC) and utilized the SURVEY procedures that account for NHANES survey multistage complex design [21].

\section{Results}

The use of medications other than the five major classes decreased from about $6.5 \%$ in $1999-2004$ to $4.6 \%$ in 2005-2008 and 3.9\% in 2009-2012. Those "other" included miscellaneous cardiovascular agents, vasodilators, vasopressors, agents for pulmonary hypertension, aldosterone receptor antagonists, and renin inhibitors; and they were not considered in this study. The use of alpha- 1 blockers increased from $4.27 \%$ in $1999-2004$ to $5.44 \%$ in $2005-2008$ and $6 \%$ in 2009-2012. Similarly, the use of central alpha2 antagonists and other centrally acting antiadrenergic agents increased from $3.58 \%$ in $1999-2004$ to $3.81 \%$ in 2005-2008 and 5.28\% in 2009-2012. This study did not examine alpha-blockers, but focused only on the use of five major classes of antihypertensive medications recommended by JNC-7 to treat hypertension in patients with CKD [7].

3.1. Prevalence of Use of Antihypertensive Medications. From 1999 to 2012, the prevalence \% (SE) of adults with CKD and
HTN who were treated for HTN increased from $55.58 \%$ (1.81) to $64.76 \%$ (1.91). Among five classes of medications, the use of ARB polytherapy increased the most: from $12.26 \%(1.44)$ to $21.56 \%(2.22)$; and the use of DIU polytherapy decreased the most: from $49 \%$ (2.55) to $42.05 \%$ (2.35); from 1999-2004 to 2009-2012. Two out of three patients with CKD and HTN received combination of classes (Table 1 ).

The prevalence of users of more than one class decreased from $65.76 \%(2.24)$ to $62.21 \%$ (3.31); however, the distributions by CKD stages for these users demonstrated increase in advanced stages, from $55.45 \%$ (2.28) to $56.20 \%$ (3.59) in stage 3 and from $7.33 \%(1.62)$ to $8.97 \%(1.85)$ in stages 4 and 5 combined, and decrease in early stages ( 1 and 2 ) from $37.21 \%$ to $34.82 \%$ (Table 1 ). The prevalence of patients was consistently greater in advanced (3) versus any early stages (1 or 2) among uses of each class. However, the increase in advanced stages was observed for ARB polytherapy and Diuretics monotherapy. The most increase in advanced stages was observed for ARB polytherapy: from $49.97 \%$ (5.61) to $70.72 \%$ (5.06) for stage 3 and from $5.7 \%$ (1.96) to $7.42 \%$ (1.94) for stages 4 and 5; while the early stages decreased from $29.58 \%$ (6.57) to $15.87 \%$ (4.38) for stage 2 and from $14.74 \%$ (5.72) to $5.98 \%$ (2.48) for stage 2 .

3.2. Antihypertensive Medications and CKD Stages: Trend Analyses. The significant increase in the odds of advanced 
TABLE 1: Age-standardized $\$$ prevalence of American adults (18 years of age or older) with hypertensive CKD treated for hypertension by five classes of medications and CKD stages: NHANES 1999-2012.

\begin{tabular}{|c|c|c|c|c|}
\hline Population & CKD & $\begin{array}{c}1999-2004 \\
\%(\mathrm{SE}) \\
\end{array}$ & $\begin{array}{c}2005-2008 \\
\%(\mathrm{SE}) \\
\end{array}$ & $\begin{array}{c}2009-2012 \\
\%(\mathrm{SE})\end{array}$ \\
\hline \multirow{5}{*}{ CKD\& HTN treated } & Total & $\begin{array}{c}N=1122 \\
55.58(1.81)\end{array}$ & $\begin{array}{c}N=916 \\
62.13(1.87)\end{array}$ & $\begin{array}{c}N=1007 \\
64.76(1.91)\end{array}$ \\
\hline & Stage 1 & $17.80(2.15)$ & $17.18(1.83)$ & $16.65(2.01)$ \\
\hline & Stage 2 & $24.81(2.43)$ & $26.64(2.36)$ & $25.06(2.68)$ \\
\hline & Stage 3 & $51.38(2.68)$ & $52.08(3.40)$ & $52.10(3.19)$ \\
\hline & Stages $4 \& 5$ & $6.00(1.34)$ & $4.09(0.78)$ & $6.17(1.02)$ \\
\hline Poly-or monotherapy & & $N=1049$ & $N=874$ & $N=968$ \\
\hline \multirow{6}{*}{$\begin{array}{l}\text { Angiotensin } \\
\text { Converting Enzyme } \\
\text { Inhibitors (ACE I) }\end{array}$} & & 462 & 395 & 424 \\
\hline & Total & $42.48(1.93)$ & 47.68 (1.68) & $40.40(2.08)$ \\
\hline & Stage 1 & $13.91(2.98)$ & $17.38(2.58)$ & $20.49(3.22)$ \\
\hline & Stage 2 & $28.61(4.46)$ & $31.59(3.58)$ & $28.40(3.38)$ \\
\hline & Stage 3 & $51.40(3.89)$ & $48.42(3.89)$ & $46.14(4.03)$ \\
\hline & Stages $4 \& 5$ & $6.08^{*}(1.89)$ & $2.60(0.70)$ & $4.96^{*}(1.79)$ \\
\hline \multirow{6}{*}{$\begin{array}{l}\text { Angiotensin Receptor } \\
\text { Blockers (ARB) }\end{array}$} & \multirow{2}{*}{ Total } & 176 & 218 & 282 \\
\hline & & $15.93(1.78)$ & $22.30(2.30)$ & $26.10(2.37)$ \\
\hline & Stage 1 & $18.28(4.76)$ & $12.13^{*}(4.04)$ & $5.83^{*}(2.24)$ \\
\hline & Stage 2 & $24.88(5.42)$ & $22.72(5.47)$ & $19.56(5.32)$ \\
\hline & Stage 3 & $52.17(5.07)$ & $59.24(6.43)$ & $66.56(5.16)$ \\
\hline & Stages $4 \& 5$ & $4.65^{*}(1.50)$ & $5.89^{*}(2.08)$ & $8.03(1.89)$ \\
\hline \multirow{6}{*}{$\beta$-blockers (BB) } & \multirow{2}{*}{ Total } & 365 & 434 & 465 \\
\hline & & $38.14(2.35)$ & $43.62(2.70)$ & $41.64(3.16)$ \\
\hline & Stage 1 & $13.07(3.13)$ & $14.41(3.76)$ & $9.29(2.27)$ \\
\hline & Stage 2 & $23.04(3.02)$ & $24.95(3.51)$ & $26.90(3.84)$ \\
\hline & Stage 3 & $55.20(3.58)$ & $53.36(4.96)$ & $54.38(4.00)$ \\
\hline & Stages $4 \& 5$ & $8.69(2.12)$ & $7.27(1.58)$ & $9.41(2.16)$ \\
\hline \multirow{6}{*}{$\begin{array}{l}\text { Calcium Channel } \\
\text { Blockers (CCB) }\end{array}$} & \multirow{2}{*}{ Total } & 427 & 332 & 339 \\
\hline & & $32.39(1.92)$ & $32.84(2.17)$ & $30.34(3.21)$ \\
\hline & Stage 1 & $14.69(3.18)$ & $16.46(3.64)$ & $20.29(4.08)$ \\
\hline & Stage 2 & $26.76(4.21)$ & $25.41(4.15)$ & $21.51(3.89)$ \\
\hline & Stage 3 & $47.83(3.82)$ & $53.15(5.71)$ & $48.53(4.69)$ \\
\hline & Stages $4 \& 5$ & $10.71(2.89)$ & $4.98(1.47)$ & $9.66(2.14)$ \\
\hline \multirow{6}{*}{ Diuretics (DIU) } & \multirow{2}{*}{ Total } & 549 & 495 & 494 \\
\hline & & $51.95(2.68)$ & $51.06(2.50)$ & $47.77(2.35)$ \\
\hline & Stage 1 & $15.21(2.53)$ & $13.86(2.87)$ & $15.34(2.90)$ \\
\hline & Stage 2 & $22.19(3.10)$ & $26.82(3.53)$ & $18.80(2.73)$ \\
\hline & Stage 3 & $58.07(3.27)$ & $54.33(4.04)$ & $58.62(3.94)$ \\
\hline & Stages $4 \& 5$ & $4.51(1.10)$ & $4.98(1.26)$ & $7.23(1.60)$ \\
\hline \multicolumn{5}{|l|}{ Polytherapy } \\
\hline \multirow{6}{*}{$\begin{array}{l}\text { Angiotensin } \\
\text { Converting Enzyme } \\
\text { Inhibitors (ACE I) }\end{array}$} & \multirow{2}{*}{ Total } & 345 & 317 & 324 \\
\hline & & $31.10(1.62)$ & $34.68(2.19)$ & $28.81(2.42)$ \\
\hline & Stage 1 & $11.49(2.45)$ & $14.85(2.42)$ & $18.46(3.73)$ \\
\hline & Stage 2 & $23.99(4.32)$ & $30.01(3.47)$ & $27.24(4.25)$ \\
\hline & Stage 3 & $55.36(4.21)$ & $51.72(4.40)$ & $48.53(3.86)$ \\
\hline & Stages $4 \& 5$ & $9.16^{*}(3.07)$ & $3.41(1.01)$ & $5.76^{*}(2.03)$ \\
\hline
\end{tabular}


TABle 1: Continued.

\begin{tabular}{|c|c|c|c|c|}
\hline Population & CKD & $\begin{array}{c}1999-2004 \\
\%(\mathrm{SE})\end{array}$ & $\begin{array}{c}2005-2008 \\
\%(\mathrm{SE})\end{array}$ & $\begin{array}{c}2009-2012 \\
\%(\mathrm{SE})\end{array}$ \\
\hline \multirow{6}{*}{$\begin{array}{l}\text { Angiotensin Receptor } \\
\text { Blockers (ARB) }\end{array}$} & \multirow{2}{*}{ Total } & 144 & 189 & 227 \\
\hline & & $12.26(1.44)$ & $18.17(2.21)$ & $21.56(2.22)$ \\
\hline & Stage 1 & $14.74^{*}(5.72)$ & $14.58^{*}(4.86)$ & $5.98^{*}(2.48)$ \\
\hline & Stage 2 & $29.58(6.57)$ & $24.02(6.03)$ & $15.87(4.38)$ \\
\hline & Stage 3 & $49.97(5.61)$ & $55.15(6.53)$ & $70.72(5.06)$ \\
\hline & Stages $4 \& 5$ & $5.70^{*}(1.96)$ & $6.24^{*}(1.87)$ & $7.42(1.94)$ \\
\hline \multirow{6}{*}{$\beta$-blockers (BB) } & \multirow{2}{*}{ Total } & 315 & 370 & 404 \\
\hline & & $32.45(2.06)$ & $36.08(2.39)$ & $34.33(2.71)$ \\
\hline & Stage 1 & $10.92(2.93)$ & $12.26^{*}(3.91)$ & $11.50(2.94)$ \\
\hline & Stage 2 & $23.47(3.37)$ & $25.75(3.65)$ & $20.72(3.90)$ \\
\hline & Stage 3 & $56.55(3.65)$ & $54.99(5.21)$ & $54.89(4.35)$ \\
\hline & Stages $4 \& 5$ & $9.06(2.17)$ & $6.99(1.78)$ & $12.87(3.28)$ \\
\hline \multirow{6}{*}{$\begin{array}{l}\text { Calcium Channel } \\
\text { Blockers (CCB) }\end{array}$} & \multirow{2}{*}{ Total } & 309 & 298 & 285 \\
\hline & & $24.72(1.91)$ & $29.81(2.22)$ & $24.64(3.10)$ \\
\hline & Stage 1 & $9.88(2.55)$ & $14.00(3.41)$ & $18.49(4.37)$ \\
\hline & Stage 2 & $26.28(4.79)$ & $25.47(4.38)$ & $19.55(4.24)$ \\
\hline & Stage 3 & $50.59(4.29)$ & $55.01(6.18)$ & $50.64(4.80)$ \\
\hline & Stages $4 \& 5$ & $13.24(3.46)$ & $5.51(1.60)$ & $11.31(2.61)$ \\
\hline \multirow{6}{*}{ Diuretics (DIU) } & \multirow{2}{*}{ Total } & 511 & 470 & 460 \\
\hline & & $49.36(2.55)$ & $46.98(2.34)$ & $42.05(2.35)$ \\
\hline & Stage 1 & $14.76(2.57)$ & $15.68(3.51)$ & $12.04(3.20)$ \\
\hline & Stage 2 & $22.09(3.19)$ & $26.32(3.98)$ & $19.00(3.14)$ \\
\hline & Stage 3 & $58.61(3.31)$ & $52.71(4.21)$ & $59.86(4.48)$ \\
\hline & Stages $4 \& 5$ & $4.53(1.16)$ & $5.28(1.34)$ & $9.09(2.43)$ \\
\hline \multicolumn{5}{|l|}{ Monotherapy } \\
\hline \multirow{6}{*}{$\begin{array}{l}\text { Angiotensin } \\
\text { Converting Enzyme } \\
\text { Inhibitors (ACE I) }\end{array}$} & \multirow{2}{*}{ Total } & 117 & 78 & 100 \\
\hline & & 11.38 (1.59) & $13.00(2.18)$ & $11.59(1.78)$ \\
\hline & Stage 1 & $16.63^{*}(5.53)$ & $21.82(4.47)$ & $24.97(5.16)$ \\
\hline & Stage 2 & $36.24(6.89)$ & $33.29(5.41)$ & $32.19(6.85)$ \\
\hline & Stage 3 & $45.40(5.76)$ & $44.49(5.70)$ & $41.60(6.72)$ \\
\hline & Stages 4 \& 5 & $1.72^{*}(1.19)$ & $0.39^{*}(0.41)$ & $1.24^{*}(0.65)$ \\
\hline \multirow{6}{*}{$\begin{array}{l}\text { Angiotensin Receptor } \\
\text { Blockers (ARB) }\end{array}$} & \multirow{2}{*}{ Total } & 32 & 29 & 55 \\
\hline & & $3.67(0.99)$ & $4.13(1.08)$ & $4.54(0.74)$ \\
\hline & Stage 1 & $27.57^{*}(9.96)$ & & $4.85^{*}(3.12)$ \\
\hline & Stage 2 & $9.41^{*}(4.24)$ & & $37.07^{*}(12.63)$ \\
\hline & Stage 3 & $62.22(10.37)$ & & 47.57 (8.88) \\
\hline & Stages $4 \& 5$ & $0.79^{*}(0.81)$ & & $10.50^{*}(5.34)$ \\
\hline \multirow{6}{*}{$\beta$-blockers (BB) } & \multirow{2}{*}{ Total } & 50 & 64 & 61 \\
\hline & & $5.69(1.36)$ & 7.55 (1.42) & $7.31(1.75)$ \\
\hline & Stage 1 & & $25.90(6.64)$ & $1.43^{*}(1.18)$ \\
\hline & Stage 2 & & $22.04^{*}(7.71)$ & $43.74(10.40)$ \\
\hline & Stage 3 & & $44.35(9.61)$ & $54.53(10.46)$ \\
\hline & Stages $4 \& 5$ & & $7.69^{*}(4.15)$ & $0.29^{*}(0.29)$ \\
\hline
\end{tabular}


TABLE 1: Continued.

\begin{tabular}{|c|c|c|c|c|}
\hline Population & CKD & $\begin{array}{c}1999-2004 \\
\%(\mathrm{SE}) \\
\end{array}$ & $\begin{array}{c}2005-2008 \\
\%(\mathrm{SE}) \\
\end{array}$ & $\begin{array}{c}2009-2012 \\
\%(\mathrm{SE}) \\
\end{array}$ \\
\hline \multirow{6}{*}{$\begin{array}{l}\text { Calcium Channel } \\
\text { Blockers (CCB) }\end{array}$} & \multirow{2}{*}{ Total } & 118 & 34 & 54 \\
\hline & & $7.67(1.21)$ & $3.03(0.71)$ & $5.70(1.29)$ \\
\hline & Stage 1 & $28.52(5.62)$ & & $27.20(7.21)$ \\
\hline & Stage 2 & $28.85(6.87)$ & & $30.38(7.91)$ \\
\hline & Stage 3 & $39.36(5.46)$ & & 39.04 (10.77) \\
\hline & Stages $4 \& 5$ & $3.26^{*}(1.28)$ & & $3.38^{*}(2.04)$ \\
\hline \multirow{6}{*}{ Diuretics (DIU) } & \multirow{2}{*}{ Total } & 38 & 25 & 34 \\
\hline & & $2.59(0.62)$ & $4.08^{*}(1.34)$ & $5.73(1.65)$ \\
\hline & Stage 1 & $20.99^{*}(8.61)$ & $6.71^{*}(4.92)$ & $29.59(6.83)$ \\
\hline & Stage 2 & $29.29^{*}(11.04)$ & $19.98^{*}(8.86)$ & $11.98^{*}(5.43)$ \\
\hline & Stage 3 & $45.03(11.72)$ & $73.31(8.34)$ & $58.41(7.14)$ \\
\hline & Stages $4 \& 5$ & $4.69^{*}(3.15)$ & 0 & 0 \\
\hline \multirow{6}{*}{$\begin{array}{l}\text { Monotherapy } \\
\text { Number of class meds } \\
=1\end{array}$} & \multirow{2}{*}{ Total } & 382 & 241 & 312 \\
\hline & & 31.69 (2.18) & $32.51(2.68)$ & $35.24(2.94)$ \\
\hline & Stage 1 & $22.71(3.83)$ & $20.64(2.06)$ & $19.11(3.37)$ \\
\hline & Stage 2 & $28.15(4.43)$ & $26.10(4.31)$ & $32.31(3.75)$ \\
\hline & Stage 3 & 46.37 (3.59) & $50.82(4.68)$ & $46.21(4.63)$ \\
\hline & Stages $4 \& 5$ & $2.76(0.83)$ & $2.43^{*}(0.97)$ & $2.35^{*}(0.81)$ \\
\hline \multirow{6}{*}{$\begin{array}{l}\text { Polytherapy } \\
\text { Number of class meds } \\
>1\end{array}$} & \multirow{2}{*}{ Total } & 667 & 633 & 656 \\
\hline & & $65.76(2.24)$ & $66.32(2.71)$ & $62.21(3.31)$ \\
\hline & Stage 1 & $13.93(2.49)$ & $14.35(2.43)$ & $14.65(2.94)$ \\
\hline & Stage 2 & $23.28(2.86)$ & $26.65(2.80)$ & $20.17(2.86)$ \\
\hline & Stage 3 & $55.45(2.82)$ & $53.97(4.13)$ & $56.20(3.59)$ \\
\hline & Stages $4 \& 5$ & $7.33(1.62)$ & $5.03(1.10)$ & $8.97(1.85)$ \\
\hline
\end{tabular}

${ }^{*}$ Potentially statistically unreliable estimates with relative standard errors $>30 \% .{ }^{\$}$ Age-standardized to the 2000 US standard population using 4 age groups.

versus early stages was observed for ARB users $(p=0.0069)$, users of ARB polytherapy ( $p=0.0023)$, and for users of polyversus monotherapy ( $p=0.0063)$; see Table 2 and Figure 2. Table 3 summarizes the prevalence of combinations of five antihypertensive classes of medication. The least prevalent was combination of ARB with ACEI therapy. Moreover, combinations of ARB with ACEI demonstrated decreased prevalence from 2005 through 2012: from 0.33\% ( $\mathrm{SE}=0.18$ ) to $0.04 \%(\mathrm{SE}=0.03)$ for $\mathrm{ARB}+\mathrm{ACEI} ; 0.57 \%(\mathrm{SE}=0.51)$ to $0.14 \%(\mathrm{SE}=0.11)$ for $\mathrm{ARB}+\mathrm{ACEI}+\mathrm{BB} ; 0.22 \%(\mathrm{SE}=0.20)$ to $0.06 \%(\mathrm{SE}=0.04)$ for $\mathrm{ARB}+\mathrm{ACEI}+\mathrm{CCB} ; 0.21 \%(\mathrm{SE}=0.12)$ to $0.03 \%(\mathrm{SE}=0.03)$ for $\mathrm{ARB}+\mathrm{ACEI}+\mathrm{DIU} ; 0.33 \%(\mathrm{SE}=$ $0.21)$ to $0.03 \%(\mathrm{SE}=0.03)$ for $\mathrm{ARB}+\mathrm{ACEI}+\mathrm{BB}+\mathrm{CCB}$; and $0.21 \%(\mathrm{SE}=0.15)$ to $0.17 \%(\mathrm{SE}=0.11)$ for $\mathrm{ARB}+\mathrm{ACEI}+\mathrm{BB}$ + DIU. Increased prevalence of medication combinations was observed only for ARB + ACEI + CCB + DIU from $0.08 \%$ (SE $=0.06)$ to $0.14 \%(\mathrm{SE}=0.08)$ and for $\mathrm{ARB}+\mathrm{ACEI}+\mathrm{CCB}+$ $\mathrm{DIU}+\mathrm{BB}$ from $0.17 \%(\mathrm{SE}=0.10)$ to $0.49 \%(0.24)$ from 2005 through 2012. The most prevalent combinations with ARB were ARB + DIU and ARB + DIU + BB with the prevalence above $4 \%$ from 2005 through 2012. During the same period of time, the use of combinations with ARB increased more than
$2 \%$ for $\mathrm{ARB}+\mathrm{BB}+\mathrm{CCB}$ : from $0.40 \%(\mathrm{SE}=0.14)$ to $2.29 \%$ $(\mathrm{SE}=0.88)$. The other combination with $\mathrm{ARB}$ was above $1 \%$ and increased from $1.11 \%(\mathrm{SE}=0.21)$ to $1.92 \%(\mathrm{SE}=0.76)$ for $\mathrm{ARB}+\mathrm{BB}$, from $1.81 \%(\mathrm{SE}=0.61)$ to $2.88 \%(\mathrm{SE}=0.81)$ for $\mathrm{ARB}+\mathrm{CCB}+\mathrm{DIU}$, and from $1.67 \%(\mathrm{SE}=0.61)$ to $1.88 \%(\mathrm{SE}$ $=0.52)$ for $\mathrm{ARB}+\mathrm{BB}+\mathrm{CCB}+\mathrm{DIU}$ and slightly decreased from $1.88 \%(\mathrm{SE}=0.64)$ to $1.82 \%(\mathrm{SE}=1.12)$ for $\mathrm{ARB}+\mathrm{CCB}$. None of the combinations of classes of medications with ARB demonstrated significant change in the relationships between advanced versus earlier stages of CKD from 1999 through 2012, except ARB + CCB ( $p=0.0065)$. However, complete analysis cannot be performed, particularly for ARB combinations with ACEI due to small sample sizes.

The exploratory analyses were conducted with level of significance $\alpha / 15=0.0033$ (Bonferroni correction for $\alpha=$ $0.05)$ where 15 is the number of analyses. The same models stratified by subgroups demonstrated significant increase in odds of advanced versus early stages of CKD for: (1) ARB users among patients with albuminuria $(p=0.0031)$ and (2) users of more than one class of medications with albuminuria $(p=0.002)$; table is not present. 
TABLE 2: Adjusted ${ }^{\# \#}$ odds ratios (95\% CI) for advanced (3, 4, and 5) versus early (1 and 2) stages of CKD by use of each class of medication.

\begin{tabular}{|c|c|c|c|c|}
\hline Model: class med $\$$ & $\begin{array}{c}1999-2004 \\
\text { odds ratio } \\
(95 \% \text { CI })\end{array}$ & $\begin{array}{c}2005-2008 \\
\text { odds ratio } \\
(95 \% \text { CI })\end{array}$ & $\begin{array}{c}2009-2012 \\
\text { odds ratio } \\
(95 \% \text { CI })\end{array}$ & $p^{\#}$ \\
\hline \multicolumn{5}{|l|}{$A C E I$} \\
\hline Overall & $1.08(0.69-1.70)$ & $0.85(0.63-1.14)$ & $0.67(0.43-1.04)$ & 0.1574 \\
\hline Polytherapy & $0.93(0.57-1.52)$ & $0.83(0.60-1.15)$ & $0.74(0.46-1.20)$ & 0.5417 \\
\hline Monotherapy & $1.59(0.91-2.80)$ & $1.06(0.65-1.73)$ & $0.70(0.33-1.48)$ & 0.0713 \\
\hline \multicolumn{5}{|l|}{$A R B$} \\
\hline Overall & $0.67(0.36-1.23)$ & $1.30(0.87-1.94)$ & $2.52(1.32-4.80)$ & 0.0069 \\
\hline Polytherapy & $0.55(0.29-1.03)$ & $1.20(0.80-1.80)$ & $2.63(1.37-5.07)$ & 0.0023 \\
\hline Monotherapy & $1.97(0.63-6.18)$ & $1.66(0.83-3.31)$ & $1.39(0.56-3.46)$ & 0.6575 \\
\hline \multicolumn{5}{|l|}{$B B$} \\
\hline Overall & $1.05(0.71-1.57)$ & $1.19(0.87-1.61)$ & $1.34(0.82-2.17)$ & 0.4637 \\
\hline Polytherapy & $1.05(0.67-1.65)$ & $1.23(0.89-1.69)$ & $1.43(0.91-2.27)$ & 0.3490 \\
\hline Monotherapy & $0.98(0.43-2.28)$ & $0.88(0.47-1.64)$ & $0.78(0.29-2.13)$ & 0.7414 \\
\hline \multicolumn{5}{|l|}{$C C B$} \\
\hline Overall & $1.28(0.85-1.93)$ & $1.17(0.86-1.59)$ & $1.08(0.67-1.72)$ & 0.5953 \\
\hline Polytherapy & $1.14(0.73-1.78)$ & $1.22(0.88-1.70)$ & $1.31(0.77-2.22)$ & 0.7036 \\
\hline Monotherapy & $1.24(0.63-2.46)$ & $0.78(0.45-1.36)$ & $0.49(0.24-1.02)$ & 0.0359 \\
\hline \multicolumn{5}{|l|}{$D I U$} \\
\hline Overall & $1.27(0.81-2.00)$ & $1.49(1.13-1.98)$ & $1.76(1.16-2.67)$ & 0.3344 \\
\hline Polytherapy & $1.37(0.88-2.13)$ & $1.65(1.24-2.20)$ & $1.99(1.29-3.06)$ & 0.2665 \\
\hline Monotherapy & $0.31(0.11-0.91)$ & $0.30(0.12-0.73)$ & $0.29(0.06-1.29)$ & 0.9288 \\
\hline \multicolumn{5}{|c|}{$\begin{array}{l}\text { Number of Classes med }>1 \text { (polytherapy) } \\
\text { versus }=1 \text { (monotherapy) }\end{array}$} \\
\hline Combination & $1.12(0.74-1.70)$ & $1.73(1.30-2.30)$ & $2.67(1.74-4.09)$ & 0.0063 \\
\hline
\end{tabular}

\section{Discussion}

The major finding is that there was significant increase in this relationship for the users of ARB polytherapy $(p=0.0023)$ from 1999 through 2012. The trend can be explained by the increase in prevalence of advanced stages CKD and decrease or stable prevalence in early stages among users of ARB polytherapy. Such increase in advanced CKD stages may have been explained by use of ARB combinations with ACEI that was particularly warned by JNC-7, but those combinations were least prevalent and decreased during the investigated time period. Possibly, change in the relationship was driven by ARB combinations with other classes, particularly with CCB where odds of advanced stages significantly increased compared to the odds of earlier stages, from 1999 through 2012. A significant increase in advanced stages was observed for ARB users among people with albuminuria. The results suggest that increase in ARB polytherapy may have increased advanced stages of CKD among American adults, particularly among people with albuminuria.

In this study for American adults 18 years or older with CKD, the changes in age-standardized prevalence were from $55.58 \%$ to $64.76 \%$, from $1999-2004$ to $2009-2012$ (Table 1).
$\mathrm{Gu}$ et al. reported similar significant $(p=0.03)$ increase in unadjusted prevalence of users of multiple antihypertensive drugs in this population of adults with CKD from $54.2 \%$ (3.5) to $65.6 \%$ (3.0), from 2001-2002 to 2009-2010 [22]. But the prevalence by five classes of medications reported by $\mathrm{Gu}$ et al. was smaller to that reported in this study that can be explained by having population of adults with CKD as comorbid condition in this study. For example, in the $\mathrm{Gu}$ et al. study the change in prevalence of ARB users went from $10.5 \%$ (1.1) to $22.2 \%$ (1.6) for poly- or monotherapy, from $7.6 \%$ (0.9) to $16.1 \%$ (1.1) for polytherapy, and from $3.0 \%(0.4)$ to 4.9\% (0.7) for monotherapy: NHANES from 2001-2002 to 2009-2010 [22]. In the present study, the increase in ARB users among hypertensive adults with CKD went from $15.93 \%$ (1.78) to $26.1 \%$ (2.37) for poly- or monotherapy, from $12.26 \%$ (1.44) to $21.56 \%$ (2.22) for polytherapy, and from $3.67 \%$ (0.99) to $4.54 \%$ (0.74) for monotherapy: NHANES from 1999-2004 to 2009-2012. The prevalence of advanced stages for the users of ARBs polytherapy increased from about $49.97 \%$ (5.61) to $70.72 \%$ (5.06) in stage 3 and from $5.70 \%$ (1.96) to $7.42 \%(1.94)$ in stages 4 and 5 combined, while the early stages decreased about half as much. The strength of the association suggests that the aggressive treatment of patients with CKD by ARB 


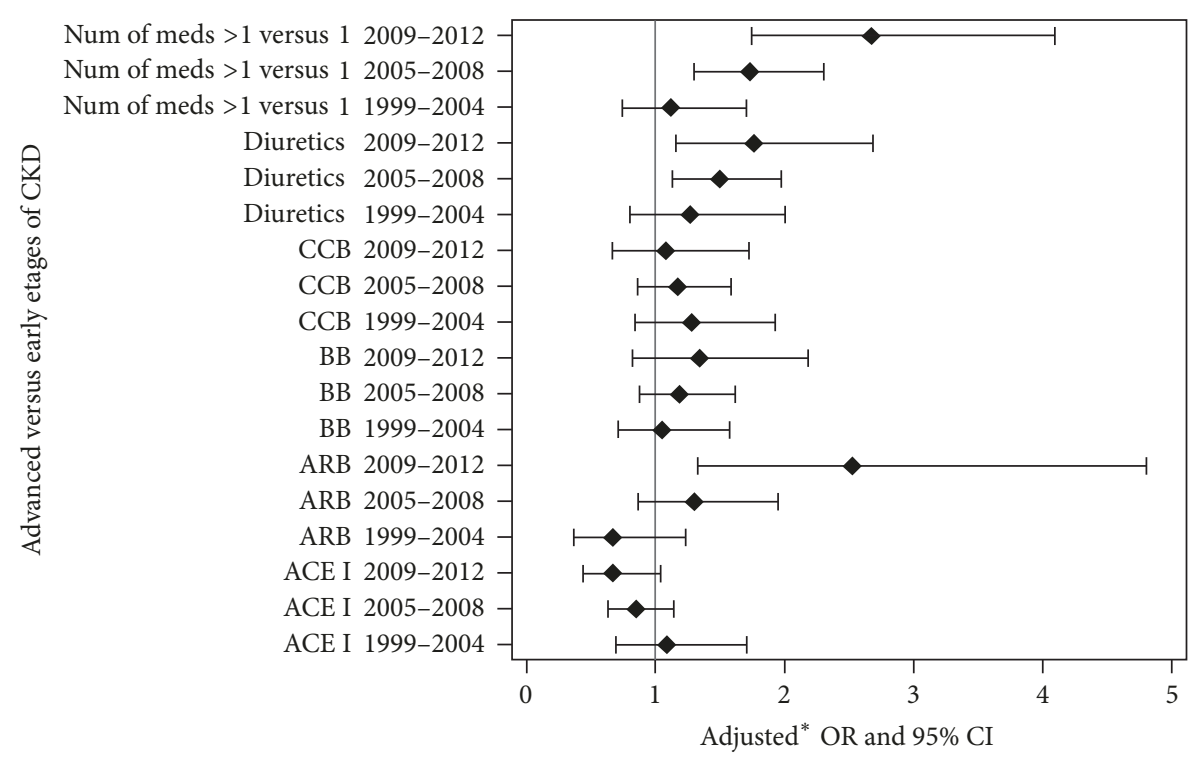

*Adjusted for age, gender, race/ethnicity, body mass index, smoking status, diabetes, albuminuria, and use of other classes

Figure 2: Forest-plots of adjusted ${ }^{*}$ odds ratios (95\% CI) for advanced $(3,4$, and 5) versus early (1 and 2) stages of CKD by use of five classes of medication. Note. ACEI = Angiotensin Converting Enzyme Inhibitors; $\mathrm{ARB}=$ Angiotensin Receptor Blockers; $\mathrm{BB}=\beta$-blockers; $\mathrm{CCB}=$ Calcium Channel Blockers; DIU = Diuretics.

could be responsible for kidney damage and the increase in advanced stages of CKD. A first population-based ecological study in the United Kingdom demonstrated an association between the increase in hospital admission rates for acute kidney injury (AKI) and the increase in prescriptions of antihypertensive medications (ACE inhibitors/ARA) in medical practices over a 4 -year time period [15]. The authors concluded that $15 \%$ of the increase in AKI admission was potentially attributable to increased prescriptions of ACE I and ARBs [15].

The current study is the first study to the authors' knowledge that estimated changes in relationships between use of antihypertensive medications and stages of CKD for American hypertensive adults with CKD. The results are consistent with the population-based ecological study in the United Kingdom and suggest that significant increase in advanced stages of $\mathrm{CKD}$ can be potentially attributable to the treatment with $\mathrm{ARB}$ polytherapy, perhaps, damaging the kidney and increasing albuminuria. However, the temporal relationship between $\mathrm{ARB}$ therapy and increase in advanced stages of CKD cannot be established because of the crosssectional nature of this study. It is possible that increase in ARB therapy resulted from the increase in advanced stages of CKD. Prospective longitudinal studies are needed to establish proper temporal sequence of this relationship.

4.1. Limitations and Strengths. The major limitation is that cross-sectional NHANES data does not allow assessing the relationship between CKD and treatment of hypertension longitudinally within patients. Another limitation is that blood pressure was measured on a single day versus on more than two separate days as was recommended by JNC 7 guidance [7]. However, three measurements were taken that might have minimized this misclassification. Similarly, CKD was defined based on single laboratory measurements versus multiple measurements during more than three months defined by KDOQI guidance [18]. However, the bias is minimal for the estimates that were the goal of this study. NHANES is a representative sample of the noninstitutionalized US population; yet, older people or people with severe cases, such as stage 3 or 4 of CKD, might have been underreported because usually weaker populations were less likely to attend MEC examination. Combining the advanced versus early stages in the statistical analyses minimized the population selection bias. Small sample size per each stage of CKD did not allow separate analysis by each stage. The combinations of classes of antihypertensive medications cannot be fully examined due to small sample sizes. Lastly, although the relationships between antihypertension medications and CKD stages are intriguing, NHANES data does not permit analysis of prescribing practices of physicians. The strength of the study is the use of NHANES data with a large sample size, good quality control, and comprehensive data collection. The long period of consistent data collected gives change to investigate the trend in the relationships between CKD stages and treatment of HTN before and after publishing JNC-7 guidelines. The presence of major risk factors for CKD and HTN was also consistently collected that gives us confidence in the results.

\section{Conclusions}

The link between ARBs and acute kidney injures was recognized recently by KDIOGO and UK National Institute for 
TABLE 3: Age-standardized prevalence of American adults (18 years of age or older) with hypertensive CKD treated For hypertension by combination of five classes of medications: NHANES 1999-2012.

\begin{tabular}{|c|c|c|c|}
\hline Combination of classes & $\begin{array}{l}1999-2004 \\
\%(S E)\end{array}$ & $\begin{array}{l}2005-2008 \\
\%(\mathrm{SE})\end{array}$ & $\begin{array}{l}\text { 2009-2012 } \\
\%(\mathrm{SE})\end{array}$ \\
\hline ACE I + ARB & $0.12^{*}(0.09)$ & $0.33^{*}(0.18)$ & $0.04^{*}(0.03)$ \\
\hline $\mathrm{ACE} \mathrm{I}+\mathrm{BB}$ & $3.41(0.89)$ & $4.06(1.02)$ & $4.24(0.73)$ \\
\hline $\mathrm{ACE} I+\mathrm{CCB}$ & $4.59(0.86)$ & $4.39(0.88)$ & $3.24^{*}(1.22)$ \\
\hline ACE I + DIU & $10.82(1.54)$ & $6.84(1.17)$ & $7.36(1.33)$ \\
\hline $\mathrm{ARB}+\mathrm{BB}$ & $0.52^{*}(0.19)$ & $1.11(0.21)$ & $1.92^{*}(0.76)$ \\
\hline $\mathrm{ARB}+\mathrm{CCB}^{\$}$ & $0.44^{*}(0.16)$ & $1.88^{*}(0.64)$ & $1.82^{*}(1.12)$ \\
\hline $\mathrm{ARB}+\mathrm{DIU}$ & $4.22(0.91)$ & $4.31(1.05)$ & $4.64(0.85)$ \\
\hline $\mathrm{BB}+\mathrm{CCB}$ & $2.12(0.51)$ & $2.88(0.64)$ & $1.44(0.41)$ \\
\hline BB + DIUX & $12.11(1.72)$ & $5.48(0.92)$ & $5.21(1.37)$ \\
\hline CCB + DIUX & $3.85(0.69)$ & $3.28(0.96)$ & $1.91^{*}(0.82)$ \\
\hline $\mathrm{ACE} \mathrm{I}+\mathrm{ARB}+\mathrm{BB}$ & $0.00(0.00)$ & $0.57^{*}(0.51)$ & $0.14^{*}(0.11)$ \\
\hline $\mathrm{ACE} \mathrm{I}+\mathrm{ARB}+\mathrm{CCB}$ & $0.22^{*}(0.16)$ & $0.22^{*}(0.20)$ & $0.06^{*}(0.04)$ \\
\hline $\mathrm{ACE} I+\mathrm{ARB}+\mathrm{DIU}$ & $0.17^{*}(0.14)$ & $0.21^{*}(0.12)$ & $0.03^{*}(0.03)$ \\
\hline $\mathrm{ACE} \mathrm{I}+\mathrm{BB}+\mathrm{CCB}$ & $2.45^{*}(0.91)$ & $2.40^{*}(0.96)$ & $2.00(0.48)$ \\
\hline $\mathrm{ACE} \mathrm{I}+\mathrm{BB}+\mathrm{DIU}$ & $2.23(0.50)$ & $6.79(1.22)$ & $5.74(1.13)$ \\
\hline $\mathrm{ACE} I+\mathrm{CCB}+\mathrm{DIU}$ & $3.09(0.70)$ & $5.02(1.30)$ & $2.39^{*}(0.82)$ \\
\hline $\mathrm{ARB}+\mathrm{BB}+\mathrm{CCB}$ & $0.34^{*}(0.26)$ & $0.40^{*}(0.14)$ & $2.49^{*}(0.88)$ \\
\hline $\mathrm{ARB}+\mathrm{BB}+\mathrm{DIU}$ & $3.08(0.69)$ & $4.70(0.81)$ & $4.66(1.27)$ \\
\hline $\mathrm{ARB}+\mathrm{CCB}+\mathrm{DIU}$ & $1.57^{*}(0.50)$ & $1.81^{*}(0.61)$ & $2.88(0.81)$ \\
\hline $\mathrm{BB}+\mathrm{CCB}+\mathrm{DIU}$ & $2.13(0.55)$ & $2.25(0.52)$ & $1.11^{*}(0.35)$ \\
\hline $\begin{array}{l}\mathrm{ACE} \mathrm{I}+\mathrm{ARB}+\mathrm{BB}+ \\
\mathrm{CCB}\end{array}$ & $0.04^{*}(0.04)$ & $0.33^{*}(0.21)$ & $0.03^{*}(0.03)$ \\
\hline $\begin{array}{l}\mathrm{ACE} \mathrm{I}+\mathrm{ARB}+\mathrm{BB}+ \\
\mathrm{DIU}\end{array}$ & $0.35^{*}(0.19)$ & $0.21^{*}(0.15)$ & $0.17^{*}(0.11)$ \\
\hline $\begin{array}{l}\mathrm{ACE} \text { I + ARB + CCB + } \\
\text { DIU }\end{array}$ & $0.48^{*}(0.48)$ & $0.08^{*}(0.06)$ & $0.14^{*}(0.08)$ \\
\hline $\mathrm{ARB}+\mathrm{BB}+\mathrm{CCB}+\mathrm{DIU}$ & $0.31^{*}(0.12)$ & $1.67^{*}(0.61)$ & $1.88(0.52)$ \\
\hline $\begin{array}{l}\mathrm{ACEI}+\mathrm{ARB}+\mathrm{BB}+ \\
\mathrm{CCB}+\mathrm{DIU}\end{array}$ & $0.20^{*}(0.16)$ & $0.17^{*}(0.10)$ & $0.49^{*}(0.24)$ \\
\hline
\end{tabular}

$\mathrm{ACEI}=$ Angiotensin Converting Enzyme Inhibitors; $\mathrm{ARB}=$ Angiotensin Receptor Blockers; $\mathrm{BB}=\beta$-blockers; $\mathrm{CCB}=$ Calcium Channel Blockers; DIU $=$ Diuretics. ${ }^{*}$ Potentially statistically unreliable estimates with relative standard errors $>30 \% .{ }^{\$}$ The use of this combination demonstrated significant increase in odds of advanced stages of CKD compared to odd of early stages, 1999 through $2012(p=0.0065)$.

Health and Clinical Excellence (NICE) [23, 24]. The results from this study suggest that increase in treatment with ARB possibly increased the prevalence of advanced CKD stages among American adults by damaging the kidneys. However, the temporal relationship cannot be claimed from this crosssectional study. Prospective studies will be necessary to establish proper temporal sequence.

\section{Conflicts of Interest}

The authors declare that there are no conflicts of interest regarding the publication of this paper.

\section{References}

[1] "U.S. Department of Health and Human Services, Office of Disease Prevention and Health Promotion. Healthy People
2020. Washington, DC,' http://www.healthypeople.gov/2020/ default.aspx.

[2] Kidney Disease Outcomes Quality Initiative (K/DOQI), "K/DOQI clinical practice guidelines on hypertension and antihypertensive agents in chronic kidney disease," Kidney Disease Outcomes Quality Initiative (K/DOQI), vol. 43, pp. S1-S290, 2004.

[3] The National Kidney and Urologic Diseases Information Clearinghouse (NKUDIC)/ National Institute of Diabetes and Digestive and Kidney Diseases (NIDDK). NIH Publication No. 14-4572 February 2014.

[4] Annals of Internal Medicine-In the Clinic ${ }^{\circledR}$ Chronic Kidney Disease American College of Physicians 2015.

[5] A. V. Chobanian, G. L. Bakris, H. R. Black et al., "The seventh report of the joint national committee on prevention, detection, evaluation, and treatment of high blood pressure: the JNC 7 report," The Journal of the American Medical Association, vol. 289, no. 19, pp. 2560-2572, 2003. 
[6] Kidney Disease: Improving Global Outcomes (KDIGO) CKD Work Group, "KDIGO 2012 clinical practice guideline for the evaluation and management of chronic kidney disease," Kidney International Supplements, vol. 3, pp. 1-150, 2013.

[7] The Seventh Report of the Joint National Committee on Prevention, Detection, Evaluation, and Treatment of High Blood Pressure; U.S. DEPARTMENT OF HEALTH AND HUMAN SERVICES; National Institutes of Health National Heart, Lung, and Blood Institute National High Blood Pressure Education Program NIH Publication No. 04-5230; August 2004.

[8] T. H. Hostetter and M. Lising, "National kidney disease education program," Journal of the American Society of Nephrology, vol. 94, 8, pp. 114S-116S, 2002.

[9] T. F. Parker III, R. Blantz, T. Hostetter et al., "The Chronic Kidney Disease Initiative," Journal of the American Society of Nephrology, vol. 15, no. 3, pp. 708-716, 2004.

[10] S. S. Yoon, V. Burt, T. Louis, and M. D. Carroll, Hypertension among adults in the United States, 2009-2010. NCHS data brief, no 107, MD: National Center for Health Statistics, Hyattsville, Md, USA, 2012, http://www.cdc.gov/nchs/data/ databriefs/db107.pdf.

[11] B. M. Egan, Y. Zhao, and R. N. Axon, "US trends in prevalence, awareness, treatment, and control of hypertension, 1988-2008," The Journal of the American Medical Association, vol. 303, no. 20, pp. 2043-2050, 2010.

[12] T. Nwankwo, S. S. S. Yoon, R. N. Vicki Burt, and Gu. Qiuping, Hypertension Among Adults in the United States: National Health and Nutrition Examination Survey, US Department of Health and Human Services, CDC/NCHS No.133, October 2013.

[13] K. L. Ong, A. W. K. Tso, K. S. L. Lam, and B. M. Y. Cheung, "Gender difference in blood pressure control and cardiovascular risk factors in Americans with diagnosed hypertension," Hypertension, vol. 51, no. 4, pp. 1142-1148, 2008.

[14] USRDS Annual Data Report; Volumel-Chronic Kidney Disease ;Volume2-End-Stage Renal Disease; 2014.

[15] L. A. Tomlinson, G. A. Abel, A. N. Chaudhry et al., "ACE inhibitor and angiotensin receptor-II antagonist prescribing and hospital admissions with acute kidney injury: A longitudinal ecological study," PLoS ONE, vol. 8, no. 11, Article ID e78465, 2013.

[16] The SPRINT Research Group, "A randomized trial of intensive versus standard blood-pressure control," The New England Journal of Medicine, vol. 373, no. 22, pp. 2103-2116, 2015, ClinicalTrials.gov number, NCT01206062.

[17] National Center for Health Statistics, National Health and Nutrition Examination Survey (NHANES) manuals (1999-2000, 2001-2002, 2003-2004, 2005-2006, 2007-2008, 2009-2010, 2011-2012, and 2013-2014), 2013, http://www.cdc.gov/nchs/ nhanes/nhanes.

[18] National Kidney Foundation, "K/DOQI clinical practice guidelines for chronic kidney disease: evaluation, classification, and stratification," American Journal of Kidney Diseases, vol. 39, 1, pp. S1-S266, Feb 2002.

[19] M. T. Crim, S. S. Yoon, E. Ortiz et al., "National surveillance definitions for hypertension prevalence and control among adults," Circulation: Cardiovascular Quality and Outcomes, vol. 5, no. 3, pp. 343-351, 2012.

[20] American Diabetes Association, "Standards of medical care in diabetes-2010," Diabetes Care, vol. 33, supplement 1, pp. S11S61, 2010.

[21] Centers for Disease Control and Prevention, National Center for Health Statistics Division of Health and Nutrition Examination
Surveys: National Health and Nutrition Examination Survey: Analytic Guidelines, 2013, http://www.cdc.gov/nchs /nhanes/.

[22] Q. Gu, V. L. Burt, C. F. Dillon, and S. Yoon, "Trends in antihypertensive medication use and blood pressure control among United States adults with hypertension: the national health and nutrition examination survey, 2001 to 2010," Circulation, vol. 126, no. 17, pp. 2105-2114, 2012.

[23] D. C. Wheeler and G. J. Becker, "Summary of KDIGO guideline. What do we really know about management of blood pressure in patients with chronic kidney disease?" Kidney International, vol. 83, no. 3, pp. 377-383, 2013.

[24] National Institute for Health and Clinical Excellence, NICE clinical guidance. Chronic kidney disease: early identification and management of chronic kidney disease in adults in primary and secondary care. 2008. 


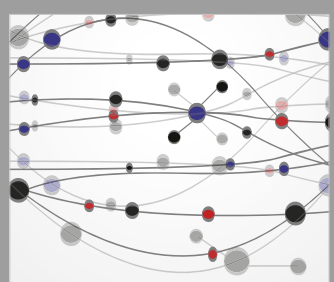

The Scientific World Journal
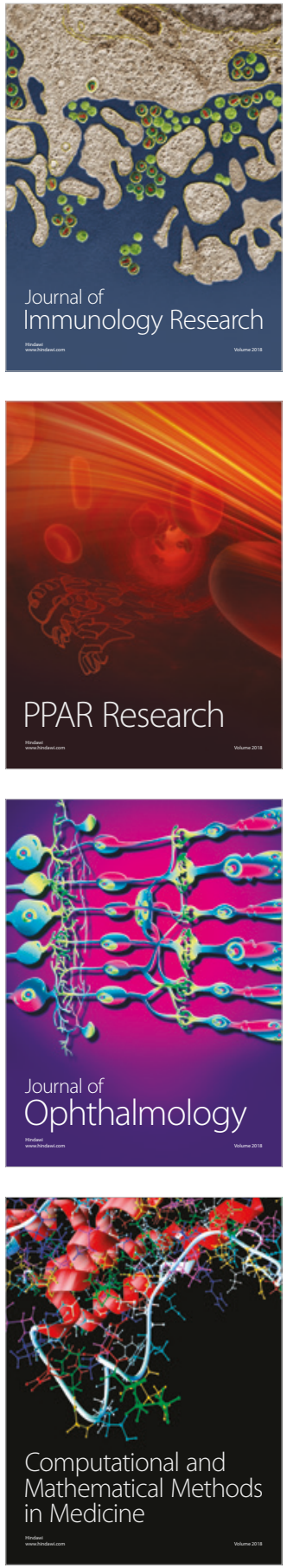

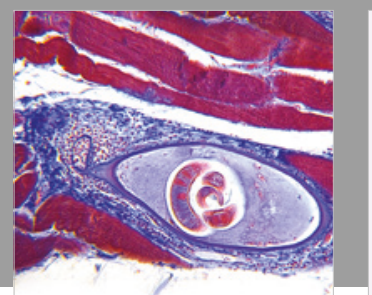

Gastroenterology Research and Practice

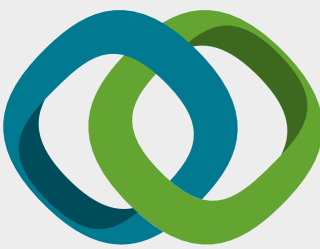

\section{Hindawi}

Submit your manuscripts at

www.hindawi.com
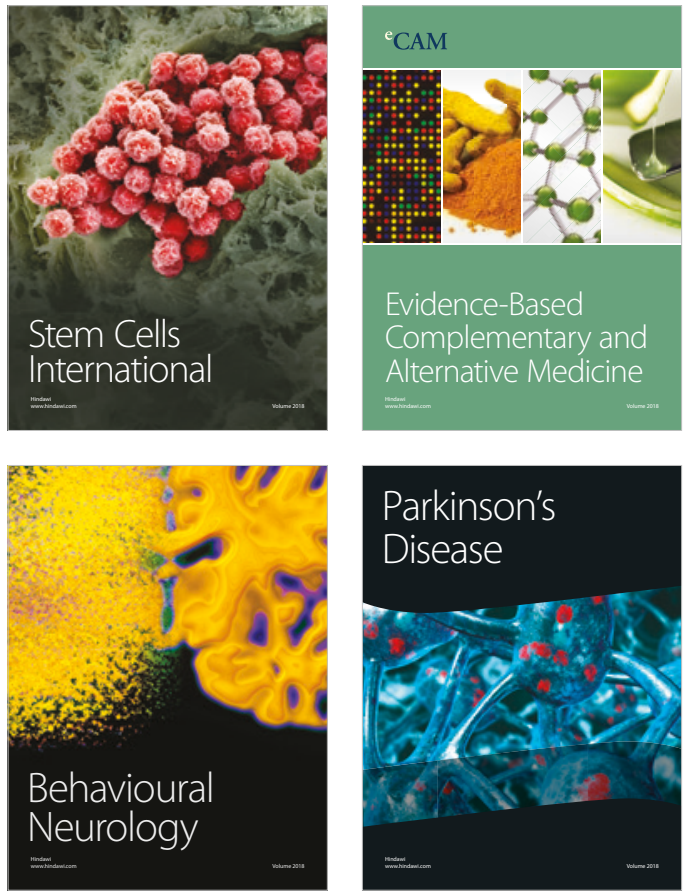

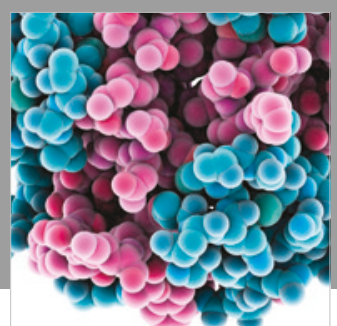

ournal of

Diabetes Research

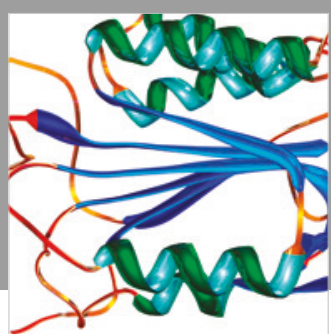

Disease Markers
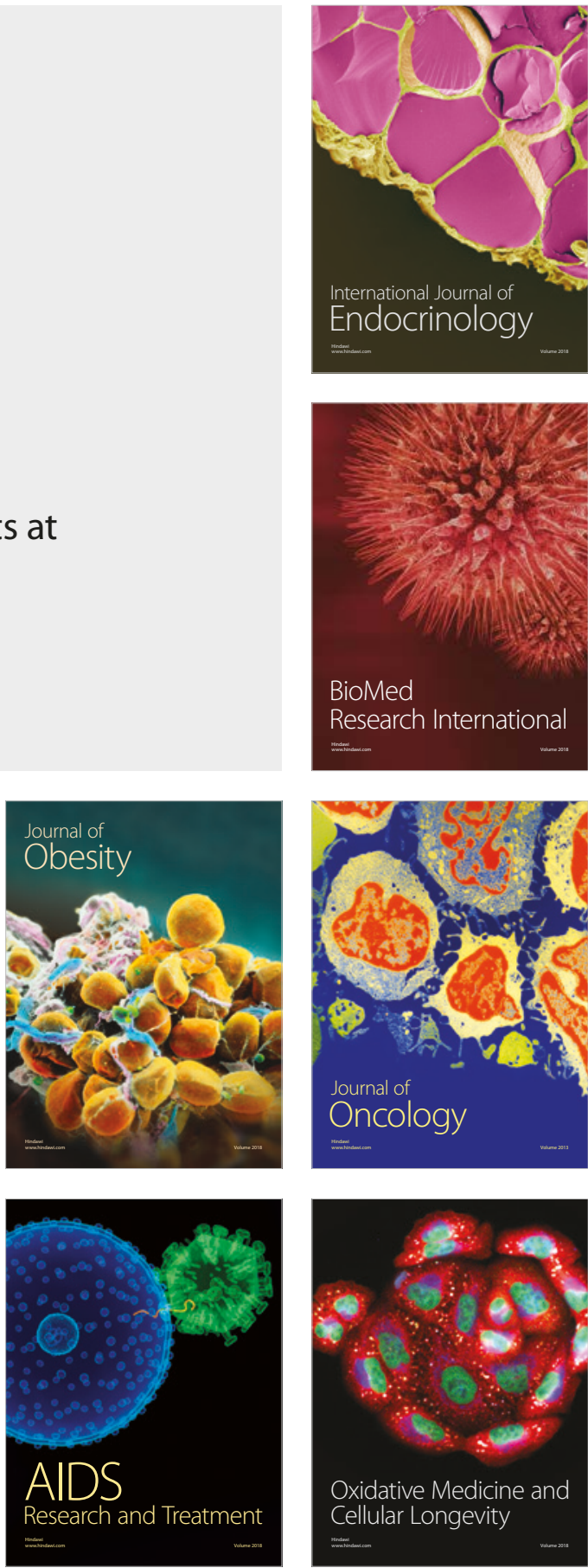